\title{
On a class of Differential Equations whose solutions satisfy Integral Equations.
}

\author{
By Professor E. T. Whittaker.
}

(Read 11th December 1914. Received 23rd December 1914.)

\section{§1. Introduction.}

The science of the solution of Differential Equations has been in great measure systematized by the aid of ideas borrowed from the Theory of Functions, the equations being classified according to the singularities possessed by their solutions. In the case of linear Differential Equations of the second order

$$
\frac{d^{2} y}{d x^{2}}+q(x) \frac{d y}{d x}+r(x) y=0,
$$

the solutions can have no singularities except at the singularities of the functions $q(x)$ and $r(x)$ (and possibly also at $x=\infty$ ): these equations may therefore be classified simply according to the number and nature of these singularities. The singularities of such a differential equation are of two kinds, regular and irregular : if $x=c$ is a regular singularity, the two independent solutions $y_{1}(x)$ and $y_{2}(x)$ in the neighbourhood of the point can be expressed in the form

$$
\begin{aligned}
& y_{1}(x)=(x-c)^{\gamma} \times \text { a series of ascending integral powers of }(x-c) \\
& y_{2}(x)=(x-c)^{\gamma^{\prime}} \times \text { a series of ascending integral powers of }(x-c),
\end{aligned}
$$
the constants $\gamma$ and $\gamma^{\prime}$ being called the exponents at this singularity. If, on the other hand, two expansions of this kind do not exist, the singularity is said to be irregular. But an irregular singularity can in most cases be regarded as formed by the coalescence or confluence of two or more regular singularities : and the problem therefore resolves itself primarily into the investigation of equations whose singularities are all regular. 
We can now classify these equations according to the number of their singularities. This number must be at least two : and the equations with only two singularities form a small and easily soluble class of no particular interest. But the equations with three regular singularities form an extensive class to which belong many equations of great importance; in particular, the differential equations satisfied by Legendre functions, by Bessel functions (a "confluent" limiting case), by the hypergeometric series, by the Hermite or parabolic-cylinder functions, by the functions $\mathrm{W}_{\mathrm{km}}(x)$, by Sonine's function $T_{m}^{m}(x)$, by Abel's function $\phi_{m}(x)$, and many others. The theory of all these functions is essentially the same, and can be derived from the fundamental general theorem that the differential equation whose singularities are at $a$ (exponents $\alpha, \alpha^{\prime}$ ), $b$ (exponents $\beta, \beta^{\prime}$ ), and $c$ (exponents $\left.\gamma, \gamma^{\prime}\right)$ is satisfied by a family of integrals of the type

$$
\begin{array}{r}
y(x)=(x-a)^{\alpha}(x-b)^{\beta}(x-c)^{\gamma} \int(s-a)^{\beta+\gamma+a^{\prime}-1}(s-b)^{\gamma+a+\beta^{\prime}-1} \\
(s-c)^{\alpha+\beta+\gamma^{\prime}-1}(x-s)^{-a-\beta-\gamma} d s \ldots \ldots
\end{array}
$$

We must now consider equations with four regular singularities. Unfortunately no theorem corresponding to (1) has been discovered for these equations : and consequently very little is known about them. I have, however, for some time past realized that in the theory of equations with four singularities, the theorem corresponding to (1) must be a honogeneous integral equation: that is, an equation of the form

$$
y(x)=\int_{a}^{b} k(x, s) y(s) d s \ldots \ldots \ldots \ldots \ldots \ldots \ldots \ldots ;
$$

and I have published the integral-equation of this kind corresponding to two differential equations of this class, namely, Mathieu's equation* and Lamés equation. $\dagger$ In the present paper I propose to consider a wider group of equations belonging to the class, and to obtain the integral-equation which is satisfied by their solutions.

* Cf. Proceedings of the International Congress of Mathenaticians, Cambridge, 1912.

+ Proceedings of the Royal Society of Edinburgh, 1914. 
\$2. Derivation of the integral-equations corresponding to differential equations of this group.

The differential equation corresponding to this schematic representation is

$$
\begin{array}{r}
z(z-b)(z-c) \frac{d^{2} y}{d z^{2}}+\left\{\frac{1}{2}(z-b)(z-c)+(1-q) z(z-c)+\frac{1}{2} z(z-b)\right\} \frac{d y}{d z} \\
+\left\{-\frac{1}{2} n\left(\frac{1}{2} n+1-q\right) z+c_{0}\right\} y=0 \ldots .
\end{array}
$$

If in this we change the independent variable by writing $z=c \sin ^{2} x$, it becomes

$\left(b-c \sin ^{2} x\right) \frac{d^{2} y}{d x^{2}}-2(1-q) \operatorname{csin} x \cos x \frac{d y}{d x}$

$$
+\left\{-n(n+2-2 q) c \sin ^{2} x+4 c_{0}\right\} y=0 . .
$$

which we shall take as the standard form of equation.

Let us see if a solution of this equation can exist of the form

$$
y(x)=\int_{0}^{2 \pi} \sin ^{n}(x+t) v(t) d t
$$

where $v(t)$ is a periodic function of $t$, of period $2 \pi$.

The condition to be satisfied is

$$
\int_{0}^{2 \pi}\left[\begin{array}{c}
\left(b-c \sin ^{2} x\right)\left\{n(n-1) \sin ^{n-2}(t+x) \cos ^{2}(t+x)-n \sin ^{n}(t+x)\right\} \\
-2(1-q) n c \sin x \cos x \sin ^{n-1}(t+x) \cos (t+x) \\
+\left\{-n(n+2-2 q) c \sin ^{2} x+4 c_{0}\right\} \sin ^{n}(t+x)
\end{array}\right] v(t) d t=0 .
$$

Replacing $x$ by $(t+x)-t$, this may be written

$$
\int_{0}^{2 \pi}\left[\begin{array}{l}
n(n-1) \sin ^{n-2}(t+x)\left(b-c \sin ^{2} t\right) \\
-\sin ^{n}(t+x)\left\{-4 c_{0}+b n^{2}+2 n(1-q) \cos ^{2} t+n(n-1) \cos 2 t\right\} \\
+2 n(n-q) \cos t \sin t \cos (x+t) \sin ^{n-1}(t+x)
\end{array}\right]^{v(t) d t=0 .}
$$

Now if $h(t)$ and $g(t)$ denote any functions of $t$ which are periodic, of period $2 \pi$, we have on integration by parts

$$
\begin{aligned}
\int_{0}^{2 \pi}(n-1) \sin ^{n-2}(t & +x) h(t) d t \\
& =\int_{0}^{2 \pi}\left\{n \sin ^{n}(t+x) h(t)-\sin ^{n-1}(t+x) \cos (t+x) h^{\prime}(t)\right\} d t
\end{aligned}
$$

and

$$
\int_{0}^{2 \pi} n \sin ^{n-1}(x+t) \cos (x+t) g(t) d t=-\int_{0}^{2 \pi} \sin ^{n}(t) g^{\prime}(t) d t
$$


Making use of these two formulae in order to raise the index of $\sin (t+x)$ to $n$ in every term of the preceding integrand, we obtain

$$
\int_{0}^{2 \pi}\left[\begin{array}{l}
\left(b-c \sin ^{2} t\right) \frac{d^{2} v}{d t^{2}}-2(n+2-q) c \sin t \cos t \frac{d v}{d t} \\
-\left\{-4 c_{0}+(n+1)(n+2-2 q) c-(n+2)(n+2-2 q) \sin ^{2} t\right\} v
\end{array}\right] \sin ^{n}(x+t) t d=0
$$

The expression (6) will therefore be a solution of the equation $(5)$, provided $v(t)$ is a periodic solution of the differential equation

$$
\begin{aligned}
& \left(b-c \sin ^{2} t\right) \frac{d^{2} v}{d t^{2}}-2(n+2-q) c \sin t \cos t \frac{d v}{d t} \\
- & \left(-4 c_{0}+(n+1)(n+2-2 q) c-(n+2)(n+2-2 q) c \sin ^{2} \iota\right\} v=0 .
\end{aligned}
$$

This is not the same differential equation as (5), but if we bring it to algebraic form by writing $c \sin ^{2} t=\zeta$, we readily find that it corresponds to the schematic representation

$$
v=P\left\{\begin{array}{cccc}
0 & b & c & \infty \\
0 & -n-1+q & 0 & \frac{1}{2} n+1-q \quad \zeta \\
\frac{1}{2} & 0 & \frac{1}{2} & \frac{1}{2} n+1
\end{array}\right\}
$$

so if we write

$$
v=(b-\zeta)^{-\frac{1}{2} n+q-1} w,
$$

the differential equation for $w$ will correspond to the schematic representation

$$
w=P\left\{\begin{array}{ccccc}
0 & b & c & \infty & \\
0 & -\frac{1}{2} n & 0 & 0 & \zeta \\
\frac{1}{2} & \frac{1}{2} n-q+1 & \frac{1}{2} & q &
\end{array}\right\} .
$$

But this is the same as the original schematic representation (3), except that the exponents at the singularities $b$ and $\infty$ have been interchanged. We can therefore reduce this to (3) by performing the homographic transformation which interchanges $b$ with $\infty$, and interchanges 0 and $c$. This transformation is

$$
\zeta=b \frac{\eta-c}{\eta-b}
$$

and if $\eta$ be defined by this equation we have therefore

$$
\boldsymbol{w}=P\left\{\begin{array}{cccc}
0 & b & c & \infty \\
0 & 0 & 0 & -\frac{1}{2} n \\
\frac{1}{2} & q & \frac{1}{2} & \frac{1}{2} n-q+1
\end{array}\right\}
$$


which is the same as (3). We may therefore (writing $\eta=c \sin ^{-s}$ ) expect that the transformation

$$
\begin{aligned}
\sin t & =\frac{\sqrt{b} \cos s}{\sqrt{b-c \sin ^{2} s}} \\
\cos t & =\frac{\sqrt{b-c} \sin s}{\sqrt{b-c \sin ^{2} s}} \\
v & =\left(b-c \sin ^{2} t\right)^{-t n+q-1} w
\end{aligned}
$$

will reduce the equation ( 7 ) to the form

$$
\left(b-c \sin ^{2} s\right) \frac{d^{2} w}{d s^{2}}-2(1-q) c \sin 8 \cos s \frac{d w}{d s}+\left\{-n(n+2-2 q) e \sin ^{2} s+4 c_{0}\right\} w=0 \ldots .
$$

which is the same equation as (5): and by actually performing the transformation we find that it is so, the value of the constant $c_{0}$ being the same in both cases.

Now $v(t)$ was a periodic solution of $(7)$ : so $w(s)$ must be a periodic solution of (9): and thus from (6) and (8) we have the result that the differential equation (5) is satisfied by the integral

$$
\int_{0}^{2 \pi}(\sqrt{b-c \sin x} \sin s+\sqrt{b} \cos x \cos s)^{n}\left(b-c \sin ^{2} s\right)^{-q} w(s) d s
$$

where $w(s)$ denotes a periodic solution of the differential equation (9).

Now this integral is evidently a periodic function of $x$, of period $2 \pi$. But since the equation (5) cannot have more than one independent periodic solution corresponding to the same value of $c_{0}$, we see that the solution represented by this integral must be (save for a constant factor) the same function of $x$ as $w(s)$ is of $s$. That is to say, the periodic solutions of the differential equation (5) are the solutions of the homogenous integral-equation

$y(x)=\lambda \int_{0}^{2 \pi}(\sqrt{b-c} \sin x \sin s+\sqrt{b} \cos x \cos s)^{n}\left(b-c \sin ^{2} s\right)^{-q} y(s) d s \ldots$

It will be seen that the constant $c_{0}$ of the differential equation does not occur in the integral-equation, while the constant $\lambda$ of the integral-equation does not occur in the differential equation. The integral-equation has a solution only when $\lambda$ has one of a certain number of values which may be denoted by $\lambda_{1}, \lambda_{2}, \lambda_{3}, \ldots:$ and 
corresponding to these values of $\lambda$ there exist solutions of the integral-equation, which we may denote by $y_{1}(x), y_{2}(x), y_{3}(x), \ldots \ldots$ The differential equation possesses a periodic solution only when $c_{0}$ has one of a certain number of values $c_{0}^{\prime}, c_{0}{ }^{\prime \prime}, c_{0}{ }^{\prime \prime \prime}, \ldots$ : and the above theorem asserts that the solutions of the differential equation corresponding to these values of $c_{0}$ are precisely the functions $y_{1}(x), y_{2}(x), y_{3}(x), \ldots$

\section{\$3. Orthogonal property of the solutions.}

The fact that the periodic solutions of (5) are the solutions of the integral-equation (10) can be applied in order to deduce properties of these solutions from the known general properties of integral-equations. For instance, we have immediately the theorem that if $y_{k}(x), y_{r}(x)$ are any two periodic solutions of (5), corresponding to the same values of $n$ and $q$ but different values of $c_{0}$, then

$$
\int_{0}^{2 \pi} y_{k}(x) y_{r}(x)\left(b-c \sin ^{2} x\right)^{-a} d x=0
$$

This relation enables us to determine the coefficients when an arbitraly function of $x$ is expanded as a series of the functions $y_{1}(x), y_{2}(x), y_{3}(x), \ldots$

\section{\$4. Verification for a particular solution.}

It is of interest to verify the result (10) for the case of one of the simplest solutions of the differential equation (5).

It is easily shown that in the case $n=2, q=\frac{3}{2}$, a periodic solution of $(5)$ is

$$
y(x)=c \sin ^{2} x-b l
$$

where $l$ denotes either root of the quadratic

$$
b l^{2}-2 b l+c=0
$$

In order to verify the theorem (10), we have therefore to show that

$$
c \sin ^{2} x-b l=\lambda \int_{0}^{2 \pi}(\sqrt{b-c} \sin x \sin s+\sqrt{b} \cos x \cos s)^{2}\left(b-c \sin ^{2} s\right)-\bar{z}\left(c \sin ^{2} s-b l\right) d s
$$

Now the integral on the right-hand side can be put in the form

$$
\int_{0}^{2 \pi}\left[\begin{array}{c}
\left(1-k^{2}\right)\left(\sin ^{2} x-\cos ^{2} x\right)(1-l) N-1 \\
+\left\{(1-l)\left[\cos ^{2} x-\left(1-k^{2}\right) \sin ^{2} x\right]-\left(1-k^{2}\right)\left(\sin ^{2} x-\cos ^{2} x\right)\right\} N-1 \\
-\left\{\cos ^{2} x-\left(1-k^{2}\right) \sin ^{2} x\right\} N
\end{array}\right]^{d s}
$$

where $k^{2}$ denotes $c / b$, and $N$ denotes $1-k^{2} \sin ^{2} s$. 
But it is known from the theory of elliptic integrals that

$$
\int_{0}^{2 \pi} N^{-\frac{s}{s}} d s=\frac{1}{1-k^{2}} \int_{0}^{2 \pi} N^{\frac{1}{2}} d s
$$

and therefore the integral can be expressed as the sum of two quantities

and

$$
\left[\left(2 \sin ^{2} x-1\right)(1-l)-1+\left(2-k^{2}\right) \sin ^{2} x\right] \int_{0}^{2 \pi} N^{\frac{1}{d}} d s
$$

$$
\left[(1-l)\left\{1-\left(2-k^{2}\right) \sin ^{2} x\right\}-\left(1-k^{2}\right)\left(2 \sin ^{2} x-1\right)\right] \int_{0}^{2 \pi} N^{-\frac{1}{2}} d s
$$

By aid of the quadratic (12), we can show that the coefficient of each of these integrals is a constant multiple of $\left(c \sin ^{2} x-b l\right)$, and this establishes the required result.

It will be observed that the verification, in this simple case, is not so straightforward as might perhaps have been expected.

\$5. A new class of functions, which in a limiting case become Mathieu functions.

There is one limiting case of the result (10) which leads to a new class of functions having somewhat remarkable properties. To obtain them, suppose that the ratio of $c$ to $b$ becomes indefinitely small and the constant $q$ indefinitely large, in such a way that the product $q c$ retains a finite value, equal to $l$ say.* Then the result (10) can be stated thus :-

The periodic solutions of the differential equation

$$
\frac{d^{2} y}{d x^{2}}+2 l \sin x \cos x \frac{d y}{d x}+\left(2 n l \sin ^{2} x+A\right) y=0
$$

(where $A$ denotes a constant) are the solutions of the homogeneous integral-equation

$$
y(x)=\lambda \int_{0}^{2 \pi} \cos ^{n}(x-s) e^{l \sin ^{2} s} y(s) d s
$$

* Two regular singularities now become confluent, and produce an irregular singularity. 
If we remove the second term in the differential equation by writing

$$
y=y_{1} e^{-\frac{1}{2} l \sin ^{2} x}
$$

it becomes (absorbing a constant into the $A$, and omitting the subseript 1 )

$$
\frac{d^{2} y}{d x^{2}}+\left\{A-(n+1) l \cos 2 x+\frac{1}{8} l^{2} \cos 4 x\right\} y=0
$$

and the periodic solutions of this differential equation are the solutions of the homogeneous integral-equation

$$
y(x)=\lambda \int_{0}^{2 \pi} \cos ^{n}(x-s) e^{\frac{1}{2} l\left(\sin ^{2} y+\sin ^{2} x\right)} y(s) d s .
$$

The remarkable property of these solutions is that they bear the same relation to the Mathieu functions $c e_{m}(x)$ and $s_{m}(x)$ that the associated Legendre functions $P_{n}^{m}(x)$ bear to the Bessel function $J_{m}(x)$. This will appear from the following properties :-

(i) Any one of these functions is expressible in a fnite form in terms of elementary functions; in fact, in the form

$$
e^{\frac{1}{2} l \sin ^{2} x} \mathbf{R}_{n}(\cos x, \sin x)
$$

where $R_{n}(\cos x, \sin x)$ denotes a homogeneous polynomial in $\cos x$ and $\sin x$ of degree $n$. (The truth of this statement is readily seen by inspection of the integral-equation).

(ii) These functions are orthogonal to each other, i.e. any pair of them $y_{r}(x)$ and $y_{k}(x)$ satisfy the condition

$$
\int_{0}^{2 \pi} y_{r}(x) y_{k}(x) d x=0
$$

(This follows from the fact that $y_{r}$ and $y_{k}$ are two distinct solutions of a homogeneous integral-equation with a symmetrical nucleus).

(iii) In the limit when $n$ tends to infinity and $l$ to zero in such a way that $n$ I remains finite, these functions become the Mathieu functions. (This is evident from the differential equation (13), which then becomes Mathieu's equation). 
The equation (13) is a case of G. W. Hill's equation in the Lunar Theory.

We may note that by taking $l$ to be zero in (13) and (14), keeping $n$ finite, we obtain the result that the solutions of the homogeneous integral-equation

$$
y(x)=\lambda \int_{0}^{2 \pi} \cos ^{n}(x-8) y(s) d s
$$

are $\cos n x, \sin n x, \cos (n-2) x, \sin (n-2) x, \cos (n-4) x, \sin (n-4) x, \ldots$, the set concluding with $\cos x, \sin x$ if $n$ is odd, and with a constant if $n$ is even. 